\title{
ISOPERIMETRIC INEQUALITIES FOR THE DIRICHLET EIGENVALUE PROBLEM*
}

\author{
BY CORNELIUS O. HORGAN** AND LEWIS T. WHEELER (Northwestern University and \\ University of Houston)
}

\begin{abstract}
Isoperimetric inequalities for the first Dirichlet eigenvalue are discussed, with application to the development of an approximate formula appropriate for thin strip domains.
\end{abstract}

1. Introduction. This paper is concerned with obtaining isoperimetric upper and lower bounds for the first Dirichlet eigenvalue, denoted by $q_{1}$, for bounded simplyconnected plane domains $D$. The inequalities discussed in Sec. 2 are isoperimetric (i.e., they reduce to equalities when the domain $D$ is a circular disk.) In Sec. 3, we pay particular attention to the case of plane strip domains (Fig. 1) whose boundaries consist of a pair of parallel curves and two straight segments meeting the curved parts orthogonally. The upper and lower bounds in this case are isoperimetric in the sense that their ratio tends to one as the thickness of the strip tends to zero. This will provide us with a means for assessing the accuracy of an approximate formula for $q_{1}$ appropriate for thin strip sections.

We refer to the extensive survey articles by Payne $[1,2]$ for a discussion of the various applications of isoperimetric inequalities. In the case of the eigenvalue $q_{1}$ under consideration here, it is shown in [2] how isoperimetric lower bounds for $q_{1}$ are of use in obtaining a priori inequalities in the Dirichlet problem for the Poisson equation. For another application, we refer to the recent paper of McLaurin [3] which describes how lower bounds for $q_{1}$ are of interest in the development of iterative numerical schemes for solution of the fundamental biharmonic boundary-value problem.

The first Dirichlet eigenvalue $q_{1}$ (see e.g. [2-7]) may be characterized by the minimum principle

$$
q_{1}=\operatorname{infimum}_{w=0 \text { on } \partial D} \frac{\int_{D}(\Delta w)^{2} d A}{\int_{\partial D}\left(\frac{\partial w}{\partial n}\right)^{2} d s},
$$

where $\partial D$ designates the boundary of $D$. Here $\Delta$ denotes the two-dimensional Laplacian and $\partial / \partial n$ is the outward normal derivative on $\partial D$. The Dirichlet eigenvalue problem [3-7] corresponding to (1.1) takes the form

$$
\Delta^{2} \varphi=0 \text { in } D,
$$

\footnotetext{
* Received August 16, 1975. This work was supported by NSF Grants ENG-75-13643 and ENG-74-01152. We are grateful to Professor L. E. Payne of Cornell University for kindly making available to us a copy of his notes [2].

** On leave from University of Houston.
} 


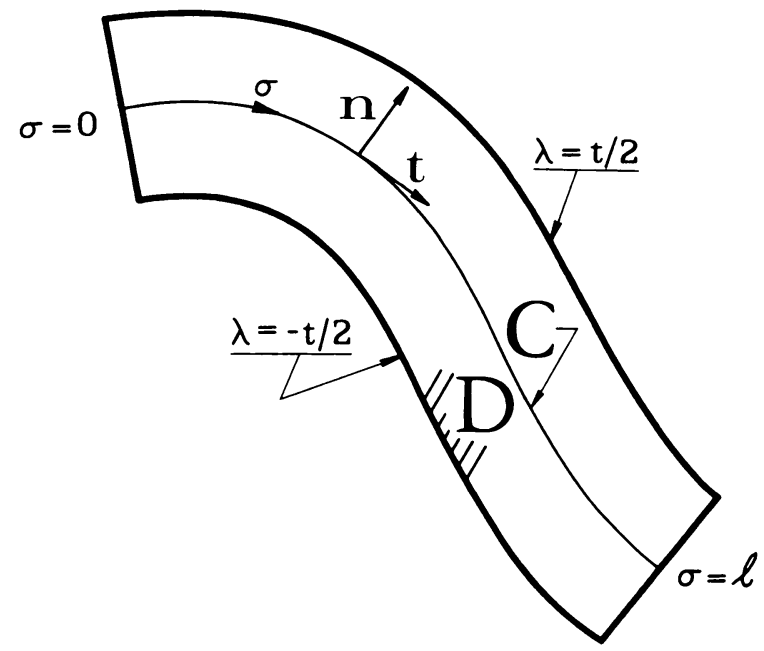

FIG. 1.

$$
\varphi=0, \quad \Delta \varphi-q_{1} \frac{\partial \varphi}{\partial n}=0 \quad \text { on } \quad \partial D .
$$

An alternative characterization for $q_{1}$ follows from the principle of duality (see [2-8]). Thus

$$
q_{1}=\operatorname{infimum}_{\Delta h=0 \text { in } D} \frac{\int_{\partial D} h^{2} d s}{\int_{D} h^{2} d A} .
$$

2. Upper and lower bounds on $q_{1}$. By using the characterization (1.4), a lower bound for $q_{1}$ has been given by Payne [2, 9] (see also [7]) in the form

$$
q_{1} \geq 2 / \tau
$$

where

$$
\tau=\max _{D+\partial D}|\nabla \Phi| .
$$

Here $\Phi$ is the Prandtl stress function for the torsion problem, and so $\Phi$ satisfies

$$
\Delta \Phi=-2 \text { in } D, \quad \Phi=0 \text { on } \partial D .
$$

The quantity $\tau$ defined in (2.2) is proportional to the maximum shear stress in torsion. It may also be written as

$$
\tau=\max _{\partial D}|\partial \Phi / \partial n| \text {. }
$$

As is pointed out in [2], explicit upper bounds for $\tau$ may be used in (2.1) to yield explicit lower bounds for $q_{1}$. 
An appropriate upper bound for $\tau$ has been obtained recently by one of the authors $[10,11]$. The upper bound derived in [10] is given by

$$
\tau \leq \rho\left(2-\kappa_{m} \rho\right)
$$

where $\rho$ is the radius of the largest possible inscribed disk in $D$ and $\kappa_{m}$ denotes the minimum curvature of $\partial D$. In [10] the curvature of $\partial D$ was assumed to be continuous at all but a finite number of points. At these points, the curvature is permitted to have a jump discontinuity appropriate to an exterior corner, re-entrant corners being excluded. The convention adopted [10] for the sign of the curvature is such that the minimum curvature $\kappa_{m}$ is negative if $D$ is not convex, whereas it is positive if $D$ is strictly convex.

Thus, on using (2.4) in (2.1), we obtain the lower bound

$$
q_{1} \geq 2 / \rho\left(2-\kappa_{m} \rho\right) \text {. }
$$

This inequality is isoperimetric by virtue of the fact that when $D$ is the interior of a disk of radius $a, q_{1}=2 / a$ and we have equality in (2.5). For the case of a convex domain $D$ with Lipschitz boundary $\partial D$, Payne [5] obtained the isoperimetric lower bound

$$
q_{1} \geq 2 \kappa_{m},
$$

with equality iff $D$ is the interior of a disk. For convex domains, (2.5) is sharper than (2.6).

An isoperimetric upper bound for $q_{1}$ has been given in [6]. Thus, on choosing $h \equiv 1$ in (1.4), one finds

$$
q_{1} \leq L / A
$$

where $L, A$ denote the perimeter and area of $D$ respectively. Again, equality holds in (2.7) when $D$ is the interior of a disk. Finally here, we note that the inequalities (2.5), (2.7) immediately provide a direct verification that $q_{1}=2 / a$ is in fact the smallest Dirichlet eigenvalue for a disk of radius $a$.

3. Thin strip domains. We consider now the case when $D$ is a strip domain, with suitable curvilinear coordinates $(\sigma, \lambda)$ introduced as shown in Fig. 1. Assuming that the midcurve $C$ admits a $\mathcal{C}^{3}$ parametrization with respect to $\sigma$ for $0 \leq \sigma \leq l$, one concludes that the curvature $\kappa(\sigma)$ is in class $\mathrm{C}^{1}[0, l]$. Furthermore, $(\sigma, \lambda)$ are coordinates in an orthogonal curvilinear system which is free of singular points, provided

$$
\bar{\kappa} t / 2<1, \text { where } \bar{\kappa}=\max _{\{0, l]}|\kappa|,
$$

and $t$ denotes the thickness of $D$.

It is readily verified that (see e.g. [11])

$$
\begin{gathered}
\rho=t / 2, \quad \kappa_{m}=-\frac{\bar{\kappa}}{1-\bar{\kappa} t / 2}, \\
A=l t, \quad L=2(l+t) .
\end{gathered}
$$

Thus, in this case, $(2.5),(2.7)$ yield the inequalities

$$
\frac{2}{t}\left(\frac{1-\bar{\kappa} t / 2}{1-\bar{\kappa} t / 4}\right) \leq q_{1} \leq \frac{2(l+t)}{l t} .
$$


These bounds have the feature that their ratio tends to one as $t$ tends to zero. For the case of an infinite strip of thickness $t$, Hersch and Payne [6] have shown that the exact value of the first Dirichlet eigenvalue is given by $\stackrel{\circ}{q}_{1}=2 / t$. The inequalities (3.4) provide a measure for assessing the accuracy in using $\dot{q}_{1}$ as an approximation to $q_{1}$ for thin strips. Thus, we write (3.4) as

$$
\frac{1-\bar{\kappa} t / 2}{1-\bar{\kappa} t / 4} \leq q_{1} / \stackrel{\circ}{q}_{1} \leq 1+t / l .
$$

From (3.5), it is clear that

$$
\lim _{t \rightarrow 0} q_{1} / \stackrel{\circ}{q}_{1}=1,
$$

for fixed $\bar{\kappa}, l$. Furthermore, (3.5) allows one to decide how small $t$ should be relative to $l$ and $\bar{\kappa}^{-1}$ in order to approximate $q_{1}$ by $\dot{q}_{1}$ to within a prescribed error.

In conclusion, it is worth mentioning that in the special case when $D$ is a rectangle $(\bar{\kappa}=$ $0)$, the inequality (3.5) reads

$$
\stackrel{\circ}{q}_{1} \leq q_{1} \leq\left(1+\frac{t}{l}\right) \stackrel{\circ}{q}_{1}
$$

Thus, in this case, the approximation $\stackrel{\circ}{q}_{1}$ is a lower bound for $q_{1}$.

\section{REFERENCES}

[1] L. E. Payne, Isoperimetric inequalities and their applications, SIAM Review 9, 453-488 (1967)

[2] L. E. Payne, Isoperimetric inequalities, maximum principles and their applications, Report of lectures given at the University, Newcastle-upon-Tyne (1972)

[3] J. W. McLaurin, A general coupled equation approach for solving the biharmonic boundary value problem, SIAM J, Numer. Anal. 11, 14-33 (1974)

[4] J. R. Kuttler and V. G. Sigillito, Inequalities for membrane and Stekloff eigenvalues, J. Math. Anal. Appl. 23, $148-160(1968)$

[5] L. E. Payne, Some isoperimetric inequalities for harmonic functions, SIAM J. Math. Anal. 1, 354-359 (1970)

[6] J. Hersch and L. E. Payne, One-dimensional auxiliary problems and a priori bounds, Abh. Math. Sem. Univ. Hamburg 36, 56-65 (1971)

[7] J. R. Kuttler, Remarks on a Stekloff eigenvalue problem, SIAM J. Numer. Anal. 9, 1-5 (1972)

[8] G. Fichera, Su un principio di dualità per talune formole maggiorazione relative alle equazioni differenziali, Atti Accad. Naz. Lincei Rend. Cl. Sci. Fis. Mat. Natur. 19, 411-418 (1955)

[9] L. E. Payne, Bounds for the maximum stress in the Saint Venant torsion problem, Indian J. Mech. Math., special issue in honor of B. Sen, Part I, 51-59 (1968)

[10] S.-L. Fu and L. Wheeler, Stress bounds for bars in torsion, J. Elasticity 3, 1-13 (1973)

[11] L. Wheeler and S.-L. Fu, Stress bounds for twisted bars of strip cross section, Int. J. Solids Struct. 10, 461-468 (1974) 